\title{
Intrusion Detection and Avoidance System
}

\author{
Manjunath R. Hudagi ${ }^{\# 1}$, Sachin A Urabinahatti ${ }^{\# 2}$,Dr.Rajkumar L. Biradar ${ }^{\# 3}$ \\ TKIET-Dept of CSE ${ }^{1}$,-Dept of CSE ${ }^{2}$, GNITS-Dept of Electronics \& Telematics Engineering ${ }^{3}$. \\ Shivaji University Maharashtra ${ }^{1}$, Shivaji University Maharashtra ${ }^{2}$,JNT University Hyderabad ${ }^{3}$. \\ mrhudagi@tkietwarana.org ${ }^{1}$, saurabinahatti@tkietwarana.org ${ }^{2}$,rajkumar_lb@yahoo.com ${ }^{3}$
}

\section{INTRODUCTION}

In today's fast world, where technology is emerging at a very fast rate and overtaking the existing system, there is a need to think over a current working system to optimize it $\&$ match it with current technology. There is a demand that the existing system would be made more and more comfortable for the user \& it should be as simple as possible. System must be useful and user friendly. So we are implementing intrusion detection and avoidance system. The detection of intrusions or intrusions attempts either manually or via software expert systems that operate on logs or other information available from the system or the network. An intrusion is a deliberate, unauthorized attempt to access or manipulate information or system and to render them unreliable or unusable [3].

\subsection{Background:}

Network security has been a very important issue, since the rising evolution of the Internet. There has been an increasing need for security systems against the external attacks from the hackers. One important type is the Intrusion Detection System (IDS). There are two major categories of the IDS:

1. Host based intrusion detection system.

2. Network based intrusion detection system.

\subsection{Application in general form in different areas}

It is used in any

1. Software Companies

2. Universities For securing the PC.

\subsection{Challenges in existing System:}

To detect and prevent intrusion in network the existing system use open source called Snort. It used rule based language combining signature, protocol and anomaly inspection method but this signature search problem is time consumption and error pruning work. Therefore some papers introduced data mining techniques into IDS.

This system uses GSM modem which helps us control our PC (system) through mobile from unauthorized user [3].

\subsection{Problem definition:}

Intrusion Detection and Avoidance System (IDAS) is software designed to detect unwanted attempts and accessing, manipulating and/or disabling computer system. These unwanted attempts can be avoided by using cell phone and GSM modem where GSM Modem act as intermediate between PC and cell phone through which the system can send alert message to authorized user.

\subsection{Scope:}

Intrusion Detection and avoidance System is a software program that looks for unusual activity on the network as well as host and reports it either to an administrator or the user of the computer on which it was detected. The system after detecting certain wellattacks gives warning message to the user which will be received on his cell phone through the GSM Modem which acts as interface between pc and cell phone.

\section{LITERATURE SURVEY}

1] Host-based intrusion detection system, ByVokorokos, L. Technical University of Ko $\hat{A}_{i}$ ice, Department of Computers and Informatics, Slovak Republic.

The paper presents intrusion detection system which informs system administrator about potential intrusion incidence in a system. The designed architecture employee's statistical method of data evaluation, that allows detection based on the knowledge of user activity deviation in the computer system from learned profile representing standard user behavior.

2] Automated Profile Vehicle Using GSM Modem, GPS and Media Processor DM462,_By-Muhammad Tahir Qadri, Syed Shafi-Uddin Qadri, Rafia Khalid and M. Yasir Khan. This paper discusses the implementation of the embedded system design to control automobile peripherals automatically through voice recognition system. Authorize user profile will be stored in the system which carries specific settings for the user. When the user wishes to drive the car, system will activate the personal settings by recognizing the user's voice. The real time snapshot of the driver is also taken using DM642 media processor and displayed on the LCD screen with 
available profile information. The smart car can also be remotely locked or unlocked using GSM modem. Another feature of navigation and tracking of car using GPS module is also included in the system. The system takes latitude and longitude positions from GPS and sends to the PC using GSM modem. The real time location of the car is further displayed on the map on PC.

3] Defending Yourself: The Role of Intrusion detection Systems, By: John McHugh, Alan Christie, and Julia Allen.

Intrusion detection systems are an important component of defensive measures protecting computer systems and networks from abuse. This article considers the role of IDSs in an organization's overall defensive posture and provides guidelines for IDS deployment, operation, and maintenance.

\section{REQUIREMENT ANALYSIS}

It is a continuous process throughout a project. A requirement is a capability to which a project outcome (product or service) should conform. It caters to functional and non functional requirement assumptions. The future scope of this project is also defined in this chapter.

In requirement analysis phase a researcher or a software developer need to find out what is the minimum requirement and the platform we must have on which the project should be developed it consist of taking consideration of hardware and software needed to develop the project, without any problem. And this phase also takes consideration of minimum requirement of system on which the project should install and work fully functionally

\subsubsection{Software Requirements:}

I. Operating System: WINDOWS- XP

II. Front End Tool: VB.NET

III. Back End Tool: Oracle $10 \mathrm{~g}$

\subsubsection{Hardware Requirements:}

I. RAM: Minimum $256 \mathrm{MB}$.

II. Hard Disk: Minimum 20 GB.

III. GSM Modem, One Mobile Handset.

\section{$>$ GSM Modem:}

GSM Modem can accept any GSM network operator SIM card and act just like a mobile phone with its own unique phone number. Advantage of using this modem will be that you can use its RS232 port to communicate and develop embedded applications. Applications like SMS Control, data transfer, remote control and logging can be developed easily. The modem can either be connected to PC Serial port directly or to any microcontroller. It can be used to send and receive SMS or make/receive voice calls. This GSM modem is a highly flexible plug and play quad band GSM modem for direct and easy integration to RS232 applications. Supports features like Voice, SMS, Data/Fax, GPRS and integrated TCP/IP stack.

- Insert SIM card: At the back end of the GSM modem there is facility to insert the SIM card.

- Connect Antenna: There is an antenna which provides range to the SIM card.

- Connect the power Supply: It required $12 \mathrm{~V}$ power supply.

- After giving the power supply to the Modem, LED will start blinking. At this stage Modem will be activated.

- AT commands can be sent to control GSM Modem.

\section{Applications:-}

- SMS based Remote Control \& Alerts

- Security Applications

Features:-

- Highly Reliable for $24 \times 7$ operation with Matched Antenna

- $\quad$ Status of Modem Indicated by LED

- Simple to Use \& Low Cost

- Quad Band Modem supports all GSM operator SIM cards.

Commands for send and receive SMS:

- For sending SMS in text Mode:

$\mathrm{AT}+\mathrm{CMGF}=1$ press enter

$\mathrm{AT}+\mathrm{CMGS}=$ "mobile number" press enter

Once The AT commands is given' $>$ ' prompt will be displayed on the screen. Type the message to send via SMS. After this, press $c t r l+Z$ to send the SMS. If the SMS sending is successful, "ok" will be displayed along with the message number.

\section{- For reading SMS in the text mode:}

$\mathrm{AT}+\mathrm{CMGF}=1$ Press enter

$\mathrm{AT}+\mathrm{CMGR}=$ no

Number (no.) is the message index number stored in the SIM card. For new SMS, URC will be received on the screen as +CMTI: SM 'no'. Use this number in the $\mathrm{AT}+\mathrm{CMGR}$ number to read the message. 


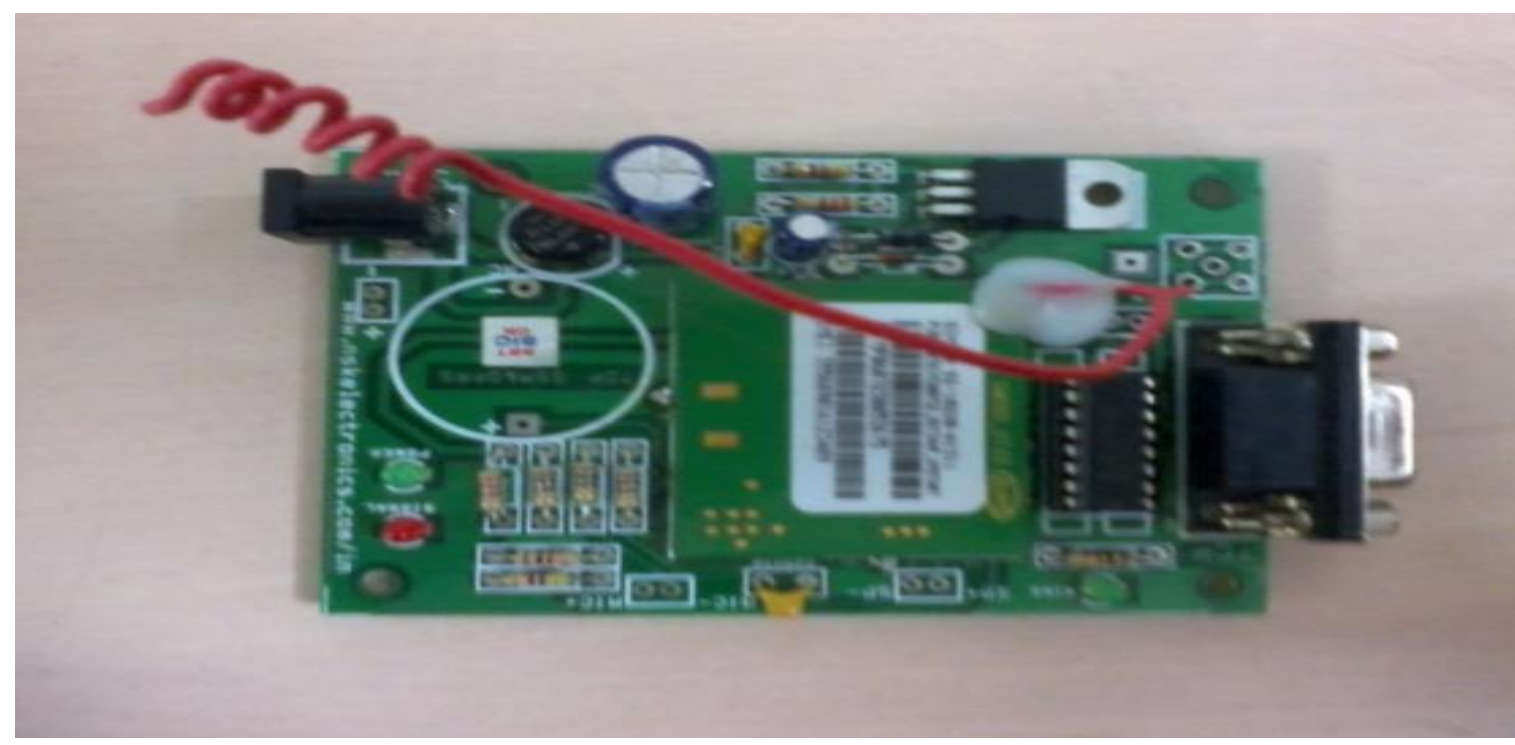

Fig 1: GSM Front Side

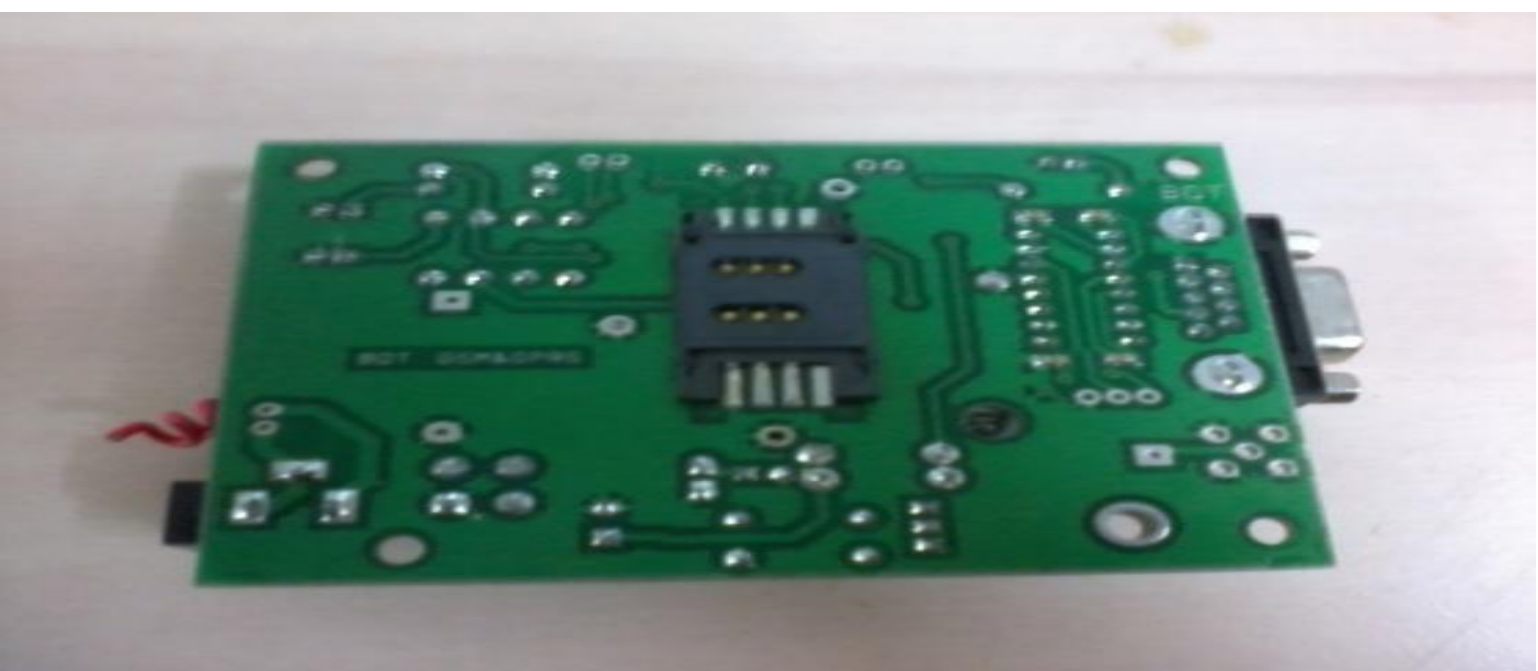

Fig 2: GSM Back Side

IV. RESULTS

4.1 Snapshot of GUI:

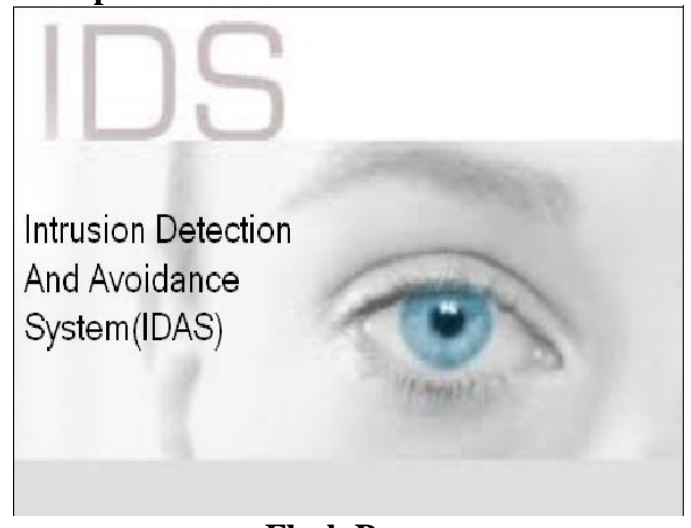

Flash Page

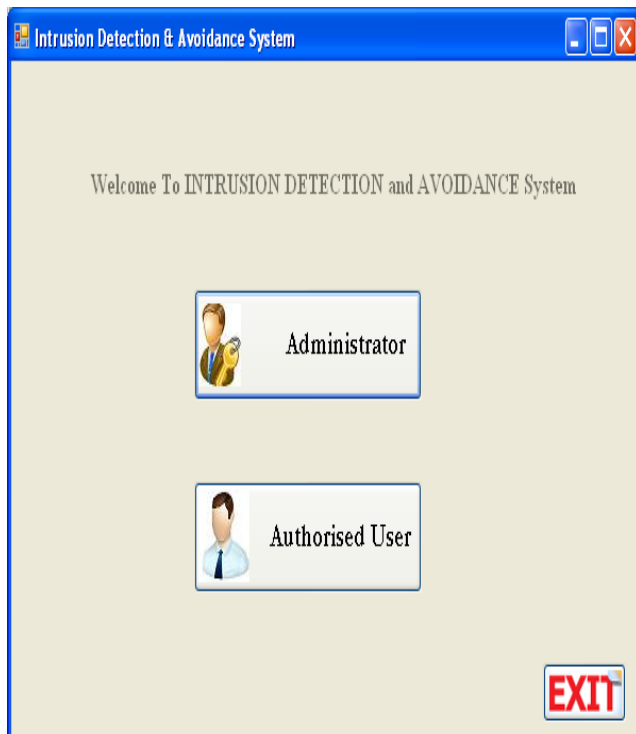

Welcome Page 


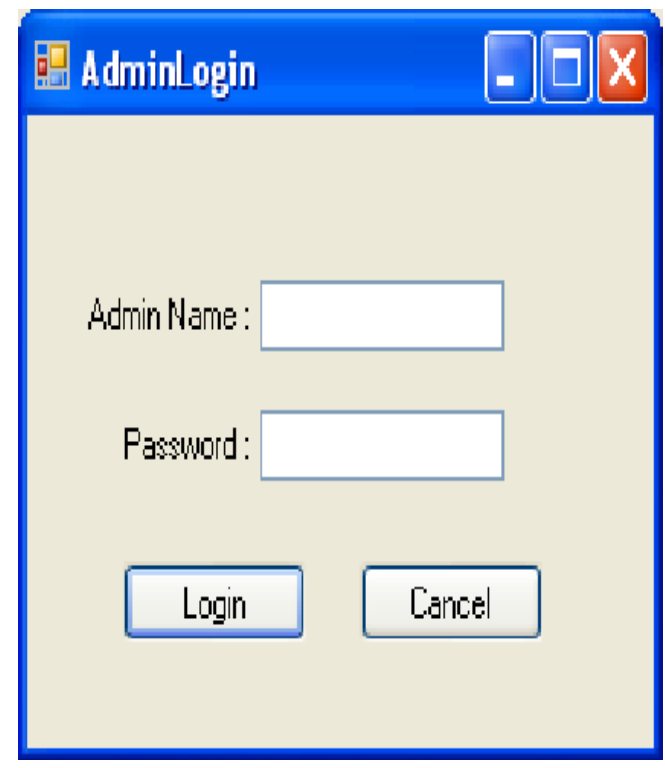

Admin Login Page

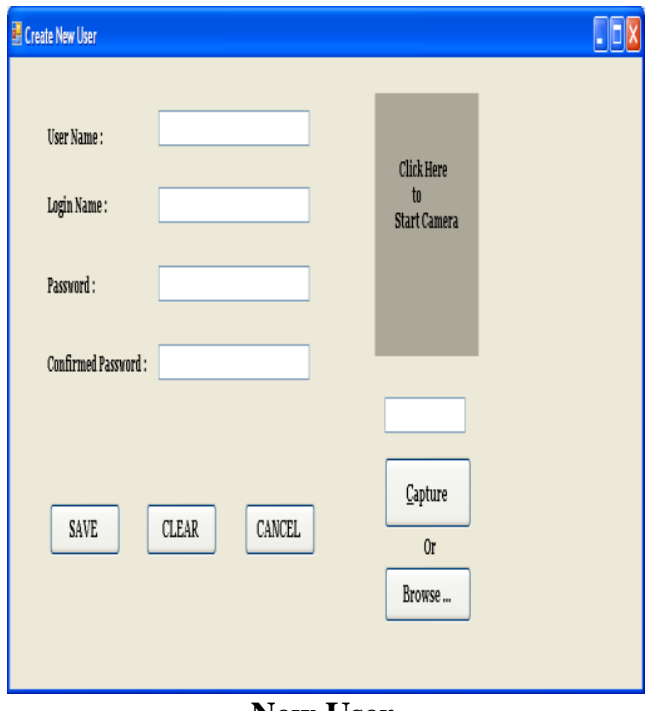

New User

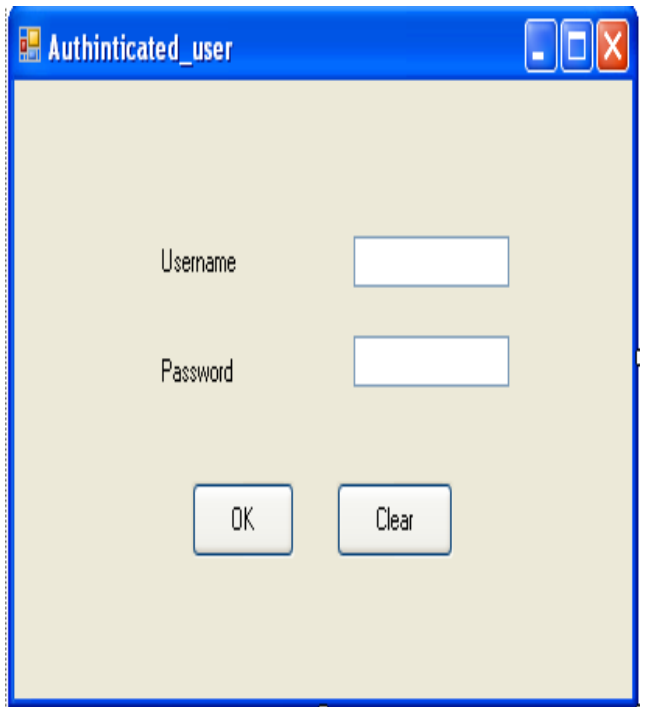

Authenticated User

\subsection{Error and Debugging Report:}

4.2.1 Database error:

The database we used is Oracle $10 \mathrm{~g}$ sometimes database is not found or connection reset error is occurred. That exception is oracle Exception.

\subsubsection{If any value exceeds than:}

The conversion of varchar value 'any value' overflowed an int column. Maximum integer value exceeded. The statement has been terminated.

\subsection{SRS Conclusion:}

\section{CONCLUSION}

By software requirement specification we conclude that there are several modules included in "Intrusion Detection and Avoidance System". The modules are like AdminLogin, CreateNewuser and AuthorizedUser.

\subsection{Design Conclusion:}

In design phase, we diagrammatically or we can say physically design the classes for easily understanding purpose. After seeing different design of web site we define our modules and final designing.

\subsection{Implementation Conclusion:}

In implementation phase, we implemented the classes, methods, events and attributes using Vb.Net language.

\subsection{Overall Conclusion:}

From a proper analysis of all aspects and points we are concluded that this project helps to detect and prevent intruders to perform malicious activity.

This project is concern with security of system. In future we can include different module which can provide more security to the system .We can also handover this software to different cooperation and organization.

\section{REFERENCES}

[1]. An integrated approach to software engineering - By Pankaj Jalote

[2]. A Novel Network Intrusion Detection System (NIDS) based on signatures Search of Data Mining, Hu Zhengbing1, 2, Li Zhitang1, Wu Junqi2.

[3]. Guide to Intrusion Detection and Prevention Systems, Karen Scarfone, Peter Mell.

[4]. GSM AT Commands Reference Guide.

[5]. The Role of Intrusion Detection System, John McHugh, Alan Christie, Julia Allen.

[6]. Intrusion Detection System. By- Wayne T Work

[7]. Automated Profile Vehicle Using GSM Modem, GPS and Media Processor DM462: 
Muhammad Tahir Qadri, Syed Shafi-Uddin Qadri, Rafia Khalid and M.Yasir Khan.

[8]. Defending Yourself: The Role of Intrusion Detection System John McHugh, Alan Christie, and Julia Allen. 